\title{
Effect of Thyroid Hormones on Adipose Tissue Flexibility
}

\author{
Lisa Volke ${ }^{a, b}$ Kerstin Krause ${ }^{a, b}$ \\ ${ }^{a}$ Medical Department III-Endocrinology, Nephrology, and Rheumatology, University of Leipzig Medical Center,

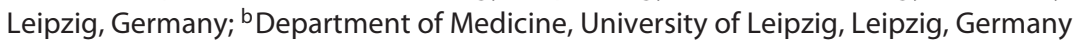

\section{Keywords}

Thyroid hormone · Browning · Beige adipocytes ·

Thermogenesis

\begin{abstract}
The recruitment and activation of energy-consuming brown adipocytes is currently considered as potential therapeutic approach to combat obesity. Thyroid hormones (TH) significantly contribute to full thermogenic capacity of brown adipocytes. A number of recent studies suggest that $\mathrm{TH}$ also induce the recruitment of brown adipocytes in white adipose depots, a process known as browning. In this review, we will summarize underlying mechanisms by which TH mediate brown adipose tissue activity and white adipose tissue browning. Furthermore, we will discuss the relevance of $\mathrm{TH}$-induced white adipose tissue browning for thermoregulation.
\end{abstract}

(c) 2020 The Author(s)

Published by S. Karger AG, Basel

\section{Thyroid Hormones and Energy Metabolism}

Thyroid hormones (TH) are main regulators of basal metabolism and are important for heat generation during shivering and nonshivering cold adaptation [1]. Changes in the thyroidal state are associated with changes in key metabolic pathways - mainly in the brain, skeletal mus- cle, liver, pancreas, and adipose tissue - that control energy expenditure (EE) and energy storage [1]. TH stimulate the basal metabolic rate, in particular through enhancement of adenosine triphosphate (ATP) production and generation and maintenance of ion gradients [1]. For instance, TH increase the production of ATP in muscle and simultaneously stimulate futile cycles involving $\mathrm{Na}^{+}$/ $\mathrm{K}^{+}$ATPase and SERCA (sarcoplasmic/endoplasmic reticulum $\mathrm{Ca}^{2+}$ ATPase), which leads to increased ATP consumption [2]. The effect of TH on EE is probably best illustrated in clinical states of hypo- or hyperthyroidism where EE can decrease or increase up to 3 times compared to baseline [3]. TH negatively regulate their own production and secretion through central mechanisms modulating the hypothalamic-pituitary-thyroid (HPT) axis via thyrotropin-releasing hormone (TRH) and subsequent thyroid-stimulating hormone (TSH) secretion. The central regulation of TH production by $\mathrm{TRH} / \mathrm{TSH}$ integrates adrenergic signaling and metabolic signals [4]. In addition to basal EE, TH are involved in adaptive thermogenesis. TH cross the blood-brain barrier and act on the hypothalamus to increase sympathetic nervous system (SNS) activation [4]. Furthermore, the TH axis can modulate the heat-generating capability of brown adipose tissue (BAT) via uncoupling protein 1 (UCP1). UCP1 dissipates the electrochemical gradient in the mitochondrial membrane that drives ATP synthesis, which leads to in-
(C) 2020 The Author(s)

Published by S. Karger AG, Basel

This is an Open Access article licensed under the Creative Commons Attribution-NonCommercial-4.0 International License (CC BY-NC) (http://www.karger.com/Services/OpenAccessLicense), applicable to the online version of the article only. Usage and distribution for commercial purposes requires written permission.
Kerstin Krause

Department of Medicine, University of Leipzig

Liebigstrasse 21

DE-04103 Leipzig (Germany)

Kerstin.Krause@medizin.uni-leipzig.de 
creased activity of the respiratory chain and heat release [5]. Collectively, this underlies the importance of TH for energy metabolism, body weight, and body temperature regulation. In humans, this paradigm is probably best reflected in hypo- or hyperthyroid patients. Hypothyroidism is associated with weight gain, whereas hyperthyroidism results in body weight reduction as a consequence of catabolic effects on muscle and adipose tissue. Changes in body weight and visceral fat mass correlate with TSH [6, 7]. Furthermore, T3 levels are directly associated with BMI and waist circumference [7]. For a detailed review of human studies on the cross talk between $\mathrm{TH}$ and adipose tissue we refer to the literature [8-10]. However, it is yet not fully clear whether changes in TSH and peripheral TH are a cause or a consequence of under- or overweight. Furthermore, changes in TSH and TH might be a reversible adaptation process in response to weight change. Thus, in a study of Reinehr et al. [11], TSH and T3 were reversibly related to body weight in patients before and after weight loss (obese patients) and weight gain (anorectic patients). However, treatment of obesity and related metabolic diseases with TH appeared to be less effective than expected [reviewed in ref. 9]. Diet-induced weight loss is commonly attenuated by the drop in resting metabolic rate due to decreased T3 levels. However, T3 administration to euthyroid obese patients to induce resting metabolic rate and favor weight loss is disputable. First, adverse side effects of TH treatment include increased heart rate, cardiac hypertrophy, muscle wasting, and reduced bone density [12]. Second, the T3-mediated increase in EE can be compensated by increased energy intake due to central stimulation of appetite. Likewise, T3-induced lipolysis is accompanied by the induction of lipogenic programs. Addressing specific $\mathrm{TH}$ actions with selective TH mimetics seems to be more suitable to achieve weight loss in obese subjects (discussed in later sections) [9].

\section{Thermogenic Flexibility of Adipocytes}

Adipose tissue is a remarkably dynamic organ that responds to environmental and internal stimuli by modulating intrinsic metabolic pathways. It mainly consists of 2 types of adipocytes, which serve opposite functions in energy balance regulation. White adipocytes, characterized by a large lipid droplet, are specialized in lipid storage and mobilization of energy in the form of free fatty acids. Brown adipocytes exhibit multiple small lipid droplets and numerous mitochondria that contain UCP1, allowing uncou- pling of oxidative phosphorylation for defense against hypothermia (Fig. 1) [5]. BAT is richly innervated by sympathetic nerves and blood vessels. Hence, thermogenic capacity can be increased by induction of proliferation and morphological changes in BAT in case of cold temperatures or high caloric diet to maintain body temperature or to get rid of excess energy [13]. As a consequence, there is much interest in activating and utilizing the thermogenic properties of brown adipocytes as a therapeutic target against obesity and other metabolic diseases. Furthermore, it has long been recognized that white adipose tissue (WAT) of cold-exposed rodents can undergo extensive remodeling into a thermogenic phenotype. The process termed "WAT browning" is marked by emergence of UCP1+ multilocular adipocytes which appear to be molecularly and functionally distinct from brown adipocytes [14]. These "beige" adipocytes occur primarily in inguinal fat and are highly inducible in response to environmental conditions and external cues, particularly chronic cold acclimatization, exercise, long-term treatment with agonists of peroxisome proliferator-activated receptor $\gamma$ (PPAR $\gamma$ ), or $\beta_{3}$-adrenergic receptor ( $\beta$ AR3), or in hypermetabolic diseases like cancer cachexia [15]. Beige adipocytes have also been observed to revert to a unilocular white adipocyte phenotype, accompanied by a loss of UCP1 expression in response to environmental warmth. However, beige capacity is preserved and reactivated under renewed cold exposure [16]. To date it is not fully clarified whether beige adipocytes occur through transdifferentiation, a direct conversion of one differentiated cell into the other without dedifferentiation, or whether the white-beige switch simply reflects beige adipocytes in an inactive (dormant) and active thermogenic state [16]. Brown and white fat depots systemically affect target tissues like liver, heart, or central nervous system by secretion of regulatory molecules termed adipokines. Besides their endocrine action, adipokines may also contribute in an autocrine and paracrine manner to EE and systemic metabolic rate via the activation of thermogenesis [17].

\section{Brown Adipocyte Thermogenesis}

\section{Peripheral Effects of TH on BAT Thermogenesis}

The tissue-specific TH action depends on transmembrane transport, intracellular activation, or inactivation by deiodinases as well as expression and activity of thyroid receptors (TR), nuclear corepressors, and nuclear coactivators, which modulate gene transcription [1]. BAT exhibits a high number of TRs similar to liver or pituitary 


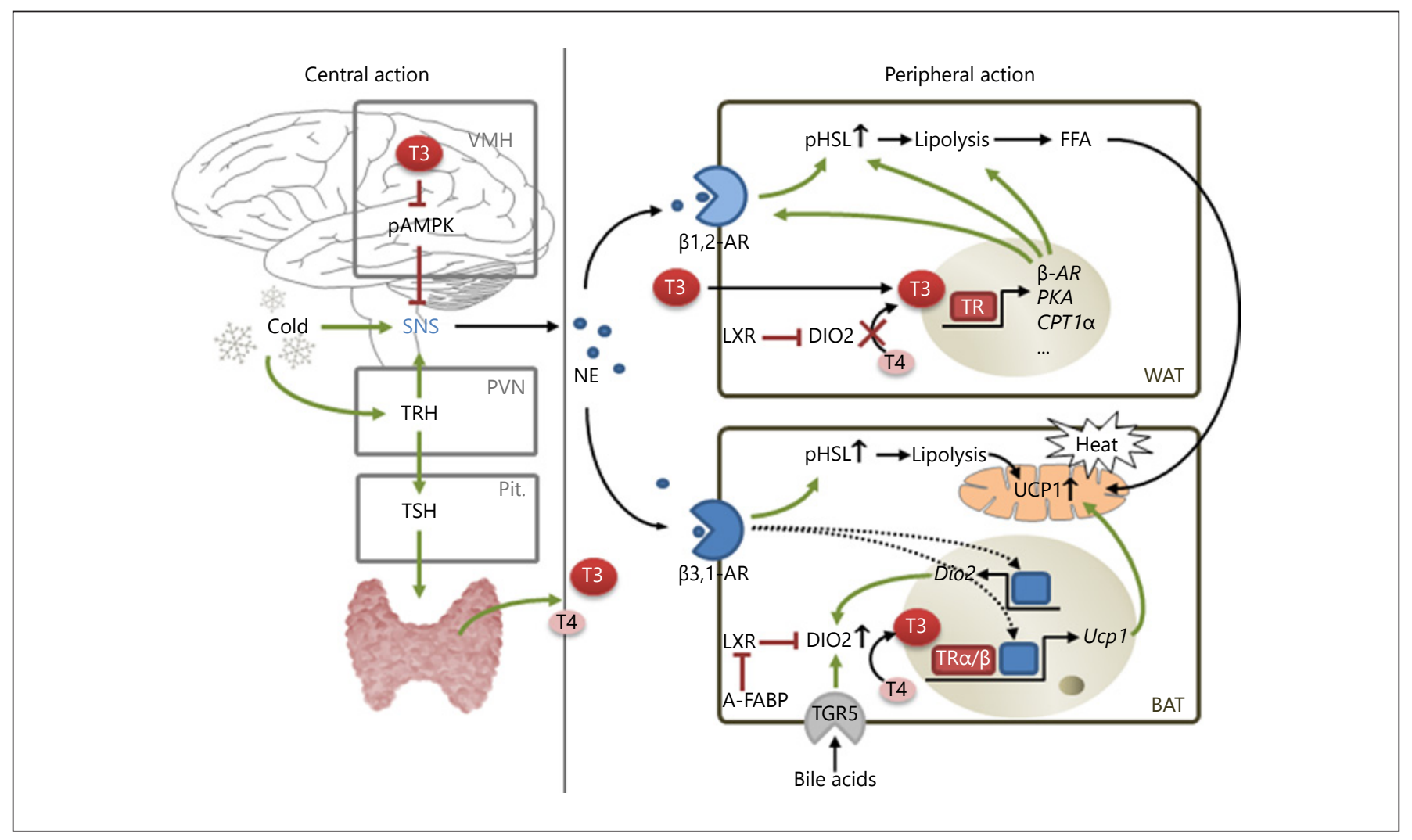

Fig. 1. Central and peripheral actions of thyroid hormones $(\mathrm{TH})$ on adipose tissues. Cold exposure activates the sympathetic nervous system (SNS) and norepinephrine (NE) release. Thyrotropinreleasing hormone (TRH)-expressing neurons in the paraventricular nucleus (PVN) are activated upon cooling, leading to augmented sympathetic outflow and increased $\mathrm{TH}$ release via the hypothalamic-pituitary-thyroid axis. T3 administration in the ventromedial nucleus of the hypothalamus (VMH) further activates SNS by inhibition of AMPK phosphorylation. NE and T3 act in a synergistic manner to regulate metabolic activity. White adipose tissue (WAT) is regulated by circulating T3, as T4-converting deiodinase 2 (DIO2) is suppressed by liver X receptor (LXR). T3 enhances NE-induced lipolysis by increasing the number of $\beta 1,2$ adrenergic receptors $(\beta 1,2-\mathrm{AR})$, postreceptor signaling, and ex-

[18]. Both isoforms are required for adequate adaptive thermogenesis in BAT. While TRa1 is needed to maintain normal adrenergic responsiveness of brown adipocytes, TR $\beta$ mediates T3-regulated Ucp1 mRNA expression [19]. However, TR $\beta$ is not essential for Ucp 1 expression in BAT, since UCP1 mRNA and protein expression levels were comparable between TR $\beta$-knock out (KO) and wild-type mice [20]. In contrast, T3-stimulated Ucp1 expression in inguinal WAT is dependent on TR $\beta$ [20]. Heat production in BAT is stimulated by the SNS but has an absolute requirement for $\mathrm{TH}$. Thus, local T3 and the catecholamine norepinephrine (NE) released from sym- pression of lipolytic enzymes as carnitine palmitoyltransferase $1 \alpha$ (CPT1 $\alpha$ ). Free fatty acids (FFA) serve as substrate and activator for thermogenesis in brown adipose tissue (BAT). Here, intracellular $\mathrm{T} 3$ is regulated by DIO2 activity, which is activated in the course of $\beta_{3}$-adrenergic activation or by bile acids via the $\mathrm{G}$ protein-coupled membrane bile acid receptor (TGR5). The inhibitor LXR is suppressed by the adipokine adipocyte fatty acid-binding protein (A-FABP). T3 and adrenergic stimulation interact to increase UCP1 gene expression and substrate delivery, promoting thermogenesis. TH receptor $\alpha(\mathrm{TR} \alpha)$ mediates synergistic effects of $\mathrm{TH}$ signaling and SNS, whereas TR $\beta$ modulates UCP1 expression. Adrenergic actions are mediated by the cAMP/protein kinase A pathway. Green, activation; red, inhibition.

pathetic nerve endings act synergistically to stimulate mitochondrial UCP1 protein expression and heat generation in BAT (Fig. 1) [21]. While T3 and NE each increase UCP1 expression 2-fold separately, there is a 20 -fold induction of UCP1 when both agents are combined [21]. Mice with global deletion of TR $\alpha 1, \mathrm{TR} \alpha 1 / \mathrm{TR} \beta$, or all TR $\alpha$ isoforms (TRa0/0) display cold intolerance associated with impaired BAT thermogenesis, based on reduced NE responses [18]. The local T3 level in brown adipocytes is controlled by deiodinase 2 (DIO2), the main enzyme converting T4 to the active T3 (Fig. 1) [22]. In fact, local action of TH during cold-induced BAT thermogenesis was 


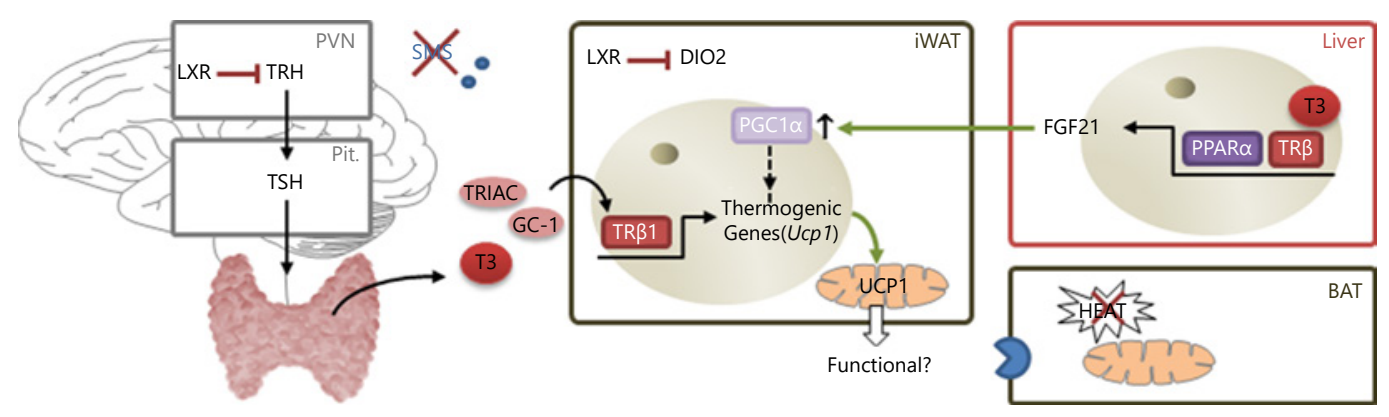

Fig. 2. Effects of TH on white adipose tissue (WAT) browning. Hyperthyroidism as well as the TR $\beta$-specific agonists triiodothyracetic acid (TRIAC) and GC-1 induce UCP1 expression in inguinal WAT (iWAT). Synergistic action is assumed for the adipokine fibroblast growth factor 21 (FGF21) via transcriptional factor PPAR $\gamma$ coactivator 1 (PGC1 $\alpha$ ). T3 directly promotes hepatic expression and secretion of FGF21 in a PPARa-dependent manner. WAT browning seems to occur independent of the sympathetic

first implied in rats in which enhanced sympathetic nerve function was found to increase DIO2 activity [22]. The importance of DIO2 for local production of T3 was demonstrated in mice with targeted Dio2 gene $\left(\mathrm{D}^{-/-}\right)$disruption. Upon cold exposure, brown adipocytes of $\mathrm{D} 2^{-/-}$ mice exhibit high lipolytic rates, based on constant NE stimulation, in order to compensate for decreased adrenergic responsiveness. This culminates in impaired BAT thermogenesis and hypothermia due to the depletion of lipid stores [23]. In addition to adrenergic stimuli, DIO2 activity can be increased as well by metabolically active molecules like insulin or bile acids [1] (Fig. 1).

\section{Central Effects of TH on BAT Thermogenesis}

The traditional concept of peripheral actions of TH on adaptive thermogenesis was challenged by several studies suggesting additional central mechanisms in hyperthyroidism. In rats, centrally administered T3 acutely activated BAT in an AMP-activated protein kinase (AMPK)dependent manner by increasing sympathetic output. This effect was blocked by a targeted expression of a mutant TR in the ventromedial nucleus of the hypothalamus [24]. Furthermore, hypothalamic TRH itself affects thermogenesis in BAT [25]. Cold exposure activates TRHexpressing neurons in the paraventricular nucleus which subsequently increase sympathetic outflow to BAT [25]. In addition, cooling induces a shift in the set point of the HPT axis, which results in increased TH release [26] (Fig. 1). nervous system (SNS) to compensate states of brown adipose tissue (BAT) inactivity. However, thermogenic activity of beige adipocytes still remains to be confirmed. Inactivation of liver X receptor (LXR) was observed to increase UCP1 expression in WAT due to increased TRH expression in the paraventricular nucleus (PVN). Further experiments are needed to distinguish between central and peripheral effects of LXR inhibition. Green, activation; red, inhibition.

\section{WAT Browning}

\section{Peripheral Effects of TH on WAT Browning}

It has been recognized that TH and several endogenous TH metabolites also induce browning of WAT. First evidence is suggested by the presence of Dio2 mRNA and activity in human preadipocytes [27] as well as by T3-induced UCP1 expression, mitochondrial biogenesis, and increased oxygen consumption in human multipotent adipose-derived stem cells [28]. The T3 metabolite 3,5-diiodo-L-thyronine (3,5-T2) was demonstrated not only to activate BAT thermogenesis in hypothyroid rats [29] but also to promote WAT browning in rats under high-fat diet at thermoneutrality, marked by reduced adipocyte size and increased mitochondrial UCP1 protein content [30]. Application of the deaminated T3 metabolite triiodo-thyracetic acid (TRIAC) to rats induced Ucp1 mRNA expression in abdominal WAT, in contrast to T3 or even cold exposure [31]. TRIAC has a higher affinity for TR $\beta 1$ than T3 in various cell types, including brown adipocytes, indicating a critical role for the TR $\beta$ isoform [31]. Accordingly, chronic administration of the synthetic, TR $\beta$-specific agonist GC-1 to $o b / o b$ mice induced marked browning of inguinal WAT (iWAT), accompanied with increased core body temperature and whole-body EE. In intrascapular BAT, gene and protein expression of UCP1 as well as ${ }^{18} \mathrm{~F}$-fluorodeoxyglucose $\left({ }^{18} \mathrm{~F}-\mathrm{FDG}\right)$ uptake was reduced, which suggests that the metabolic effects of GC-1 were 
exclusively mediated by WAT browning instead of BAT activation [32]. The direct action of GC-1 on UCP1 expression has been confirmed in primary white adipocytes, whereas GC-1 treatment in UCP1-KO mice failed to induce thermogenesis (Fig. 2) [32]. Further insights into organ-specific actions of T3 have been delivered by studies with a glucagon/T3 conjugate which selectively targets glucagon receptor-positive tissues such as liver and iWAT [33]. Comparable to systemic T3, glucagon/ T3 application led to iWAT browning in male C57BL/6J mice, evidenced by the formation of small, multilocular adipocytes as well as increased expression of $U c p 1$ and other thermogenic genes. Whereas both T3 and glucagon/T3 raised EE, only T3 enhanced food intake, locomotor activity, and body temperature. However, the degree of body weight loss was markedly higher in glucagon/T3-treated mice than in mice treated with T3 only [33]. These findings suggest that $\mathrm{TH}$ regulation of energy metabolism and thermogenesis is multilayered and involves various organs beyond adipose tissue. Another interesting aspect is that the effect of the glucagon/T3 conjugate on body weight and EE was only attenuated but not fully abolished in male UCP1-KO mice [33], which indicates an additional mechanism to promote WAT browning, such as induction of fibroblast growth factor 21 (FGF21) (Fig. 2) [34]. We recently investigated the effect of TH on WAT browning in female C57BL/6N mice. Surprisingly, we observed features of WAT browning not only in female hyperthyroid but also in hypothyroid mice compared to euthyroid controls when housed at room temperature. They showed increased thermogenic gene expression and multilocular UCP1+ adipocytes in both iWAT and gonadal WAT [35]. Induction of the thermogenic program in WAT seemed to be compensatory in response to decreased heat production according to reduced BAT activity in small-animal ${ }^{18} \mathrm{~F}$ FDG positron emission tomography (PET) measurements [35]. However, given that the genetic background essentially contributes to the complexity of metabolic responses, this should be considered for interpretation of data on adipose tissue plasticity. For instance, C57BL/6 substrains vary with respect to metabolic parameters, $\mathrm{EE}$, and activity levels due to genetic drifts, all influencing body weight and thermoregulation [36]. Especially for $\mathrm{KO}$ model generation, it is critical to avoid mixed backgrounds, if possible, and to choose suitable controls [36]. Furthermore, cold exposure studies in mice have shown that subcutaneous adipose tissue depots are more susceptible to browning than visceral adipose tissue because of greater sympathetic innervation and turnover of NE $[13,37,38]$. Also, sex hormones likely affect WAT function beyond their role in WAT distribution. Thus, it is of interest to investigate a potential thermogenic effect in both genders using suitable controls and avoiding mixed backgrounds [36].

\section{Central Effects of TH on WAT Browning}

Recent data provide evidence for a central action of $\mathrm{TH}$ on WAT browning. Central application of T3 increased UCP1 expression in BAT and browning of iWAT, associated with increased oxygen consumption and body temperature, due to a decrease in hypothalamic AMPK phosphorylation [39]. The central effects of T3 on energy metabolism were abolished in UCP1-deficient mice [39]. Interestingly, the decarboxylated and deiodinated $\mathrm{T} 3$ metabolite 3-iodothyronamine (3-T1AM) led to an anapyrexic decrease in body temperature through central induction of tail vasodilation. Compensatory activation of BAT thermogenesis, probably also WAT browning, seemed to be centrally blocked in an AMPK-dependent manner [40]. Other endogenous TH derivatives such as iodine-free thyronamine (T0AM) or 3-iodo-thyroacetic acid (TA1) revealed no or insignificant effects on thermoregulation [40].

However, the paradigm that adipose tissue is the primary target of central TH has recently been challenged by a landmark publication dissecting the contribution of central and peripheral effects of T3 on WAT browning [20]. T3-induced browning was confirmed to require $\operatorname{TR} \beta$, since browning was absent in hyperthyroid TR $\beta$ $\mathrm{KO}$ mice [20]. Interestingly, it occurred independently from SNS, as neither NE nor cAMP levels were elevated in iWAT, and the browning effect was still observed at thermoneutrality where adipose tissue is functionally denervated [20] (Fig. 2). In addition to the increased resting metabolic rate at thermoneutrality, T3-treated mice displayed increased lipid uptake and glycogen disposal in skeletal muscle. The authors concluded that the muscle is the primary source of peripheral hyperthermia in T3treated mice rather than BAT or beige adipose tissue [20]. Therefore, this study adds novel understanding to other observations showing UCP1-independent pyrexia in T4treated mice [41] and a reduced demand for adaptive thermogenesis in response to acute cold stress in T4-induced thyrotoxic rats [42]. The thermogenic response in BAT appears to decrease in states where other T3 target tissues like skeletal muscle and probably iWAT are thermogenically activated [43]. 


\section{Interaction between TH and Adipokines}

It has been appreciated that adipose tissue not only acts as a storage organ but also as an endocrine organ that produces and secrets a number of biologically active factors, so-called "adipokines." The dysregulated expression of adipokines is linked to the pathogenesis of obesity and metabolic disorders. As mentioned above, while it is known that changes in TSH/TH levels are associated with a change in body weight, only little is known about the underlying physiological pathways. A number of studies provided evidence for a central role of the adipokine leptin for the regulation of the HPT axis. In rodents, leptin promotes TRH biosynthesis through actions on TRH neurons and is important to stabilize the HPT axis during diet-induced obesity [44-46]. In a clinical context, the importance of a balanced interaction of leptin with the HPT axis is best exemplified in patients with leptin deficiency. Here the application of leptin leads to normalization of serum TH and TSH serum levels [47]. Interestingly, there is a high synchronicity between the secretion of leptin and TSH [48]. Moreover, a correlation between leptin and TSH has been reported in many cross-sectional and longitudinal studies [reviewed in ref. 10]. Conversely, TH regulate leptin mRNA and serum levels [49]. TSH treatment directly stimulated leptin secretion in-vitro [50] and in vivo most likely through TSH receptor activation [51, 52]. Altogether, these data provide compelling evidence that the connection between TH and leptin through TSH is important for body weight control and maintenance of energy balance. Furthermore, it is very likely that the interplay between $\mathrm{TH}$ and adipokines also contributes to adaptive thermogenesis. FGF21 is produced and secreted by the liver and promotes its browning effect mainly through induction of the PPAR $\gamma$ coactivator 1 (PGC1 $\alpha$ ), one of the central players of WAT browning [53]. T3 was also shown to directly induce hepatic expression of $F g f 21$ in a TR $\beta$ - and PPARa-dependent fashion (Fig. 2) [54]. The T3/glucagon conjugate study published by Finan et al. [33] revealed an enhanced hepatic expression of FGF21 along with increased WAT browning. The thermogenic effect of the glucagon/T3 conjugate was only blunted but not fully abolished, neither in FGF $21^{-/-}$nor $\mathrm{UCP} 1^{-/-}$mice, suggesting a synergistic action of T3, UCP1, and FGF21. It remains to be clarified whether this contributes to the browning of WAT observed in mice with peripheral hyperthyroidism [20]. Another adipokine implicated in adaptive thermogenesis is the adipocyte fatty acid-binding protein $(\mathrm{A}-\mathrm{FABP})$. A-FABP accelerates thermogenesis in BAT by promoting DIO2-induced $\mathrm{T} 3$ production via inhibition of the DIO2-repressing nuclear liver X receptor (LXR) a (Fig. 1) [55]. Likewise, the thermogenic response of T4 is blunted in A-FABP-KO mice but enhanced by A-FABP [55]. Moreover, mice with LXR inactivation showed increased Ucp 1 expression in iWAT, associated with an increased Trh expression in the paraventricular nucleus, more TSH-positive cells in the anterior pituitary, hypertrophy of thyrocytes, and increased circulating T3 [56] (Fig. 2). LXRa has also been shown to cell autonomously inhibit Ucp1 expression in white adipocytes [57]. Hence, future studies with neuronal-specific LXR-KO are needed to confirm the association of WAT browning and central HPT axis modulation in this model as well as to identify central LXR signaling mediators in the physiological setting.

\section{Is TH-Induced Beige Fat Thermogenically Active?}

Regardless of the observed TH-induced browning, the relevant question is whether increased beige UCP1+ adipocytes indicate thermogenic function. It is technically challenging to distinguish between brown and beige adipocyte heat production in vivo, since both are activated by adrenergic mechanisms. Determination of UCP1 expression, sympathetic activation, and mitochondrial content are useful surrogate markers of beige thermogenic activation. The current gold standard to visualize metabolically active adipose tissue is ${ }^{18} \mathrm{~F}$-FDG PET/CT [58]. However, detection of present but inactive brown and beige adipocytes is difficult. Furthermore, FDG accumulation reflects high glucose uptake and utilization and not necessarily increased thermogenic rate, as adrenergically stimulated uptake occurs even in the absence of UCP1 [59]. BAT is known to accumulate other tracers displaying fatty acid uptake $\left({ }^{18} \mathrm{~F}\right.$-fluoro-triheptadecanoic acid, ${ }^{18} \mathrm{~F}$-THA), intracellular fatty acid synthase activity $\left({ }^{11} \mathrm{C}\right.$ acetate), and adrenergic innervation ( ${ }^{123}$ I-metaiodobenzylguanidine, ${ }^{123}$ I-MIBG) [60]. It is to be clarified whether these techniques are also suitable to image thermogenic activation of beige adipocytes.

The contribution of $\mathrm{TH}$-induced WAT browning to an elevated body temperature was recently investigated in the above-mentioned study of Johann et al. [20]. In hyperthyroid mice, neither ${ }^{18} \mathrm{~F}$-FDG nor lipid uptake was elevated in iWAT although UCP1 levels were increased [20]. Moreover, even T3-treated UCP1-KO mice exhibit WAT browning associated with an equivalent increase in body temperature compared to T3-treated wild-type mice. Finally, the T3 effect on WAT browning was dem- 
onstrated to be SNS independent, as it sustained at thermoneutrality [20]. To date, it is still unclear which factors finally contribute to browning in hyperthyroid mice, although FGF-21 or glucagon are discussed as promising candidates [61]. Anyway, the study underscores the fact that the mere presence of UCP1 is insufficient to draw conclusions about thermogenic activation and therapeutic potential of browning agents.

\section{Conclusion}

The concept of central and peripheral actions of TH on BAT thermogenesis is well established. Recent findings suggest an additional regulatory role of $\mathrm{TH}$ for the induction of UCP1+ beige adipocytes in white adipose depots. Even though these data appear promising in regard of therapeutic approaches, thermogenic activity of beige adipocytes remains questionable, since several studies indicate that UCP1 expression in beige adipocytes not necessarily associates with metabolic activity. There are many issues to be answered: What is the relevance of beige adipocytes for thermoregulation in hypothyroid mice? To which extent is the coordination between central and peripheral pathways affected by dynamic changes in $\mathrm{TH}$ serum levels? How is this associated with differential activation of thermogenic tissues, e.g., BAT, WAT, and skeletal muscle, and which other factors mediate the effects $\mathrm{TH}$ on body weight control and maintenance of energy balance?

\section{Acknowledgments}

The authors thank Juliane Weiner for critical review of this manuscript.

\section{Statement of Ethics}

Does not apply.

\section{Disclosure Statement}

There is nothing to disclose.

\section{Funding Sources}

This work was supported by grants from the German Research Foundation (KR 4258/3-1 and KR 4258/1-1 SPP1629 "Thyroid TransAct").

\section{Author Contributions}

L.V. searched data for the article and wrote the article. K.K made substantial contributions to the structure and discussion of the content and contributed to the writing and editing of the article.

\section{References}

1 Mullur R, Liu YY, Brent GA. Thyroid hormone regulation of metabolism. Physiol Rev. 2014 Apr;94(2):355-82.

$2 \mathrm{Yu} \mathrm{F}$, Göthe S, Wikström L, Forrest D, Vennström B, Larsson L. Effects of thyroid hormone receptor gene disruption on myosin isoform expression in mouse skeletal muscles. Am J Physiol Regul Integr Comp Physiol. 2000 Jun;278(6):R1545-54.

3 Bianco AC, McAninch EA. The role of thyroid hormone and brown adipose tissue in energy homoeostasis. Lancet Diabetes Endocrinol. 2013 Nov; 1(3):250-8.

4 López M, Alvarez CV, Nogueiras R, Diéguez C. Energy balance regulation by thyroid hormones at central level. Trends Mol Med. 2013 Jul;19(7):418-27.

5 Cannon B, Nedergaard J. Brown adipose tissue: function and physiological significance. Physiol Rev. 2004 Jan;84(1):277-359.
6 Muscogiuri G, Sorice GP, Mezza T, Prioletta A, Lassandro AP, Pirronti T, et al. High-normal TSH values in obesity: is it insulin resistance or adipose tissue's guilt? Obesity (Silver Spring). 2013 Jan;21(1):101-6.

7 De Pergola G, Ciampolillo A, Paolotti S, Trerotoli P, Giorgino R. Free triiodothyronine and thyroid stimulating hormone are directly associated with waist circumference, independently of insulin resistance, metabolic parameters and blood pressure in overweight and obese women. Clin Endocrinol (Oxf). 2007 Aug;67(2):265-9.

8 Iwen KA, Schröder E, Brabant G. Thyroid hormones and the metabolic syndrome. Eur Thyroid J. 2013 Jun;2(2):83-92.

9 Santini F, Marzullo P, Rotondi M, Ceccarini G, Pagano L, Ippolito S, et al. Mechanisms in endocrinology: the crosstalk between thyroid gland and adipose tissue: signal integration in health and disease. Eur J Endocrinol. 2014 Oct;171(4):R137-52.
10 Delitala AP, Fanciulli G, Pes GM, Maioli M, Delitala G. Thyroid Hormones, Metabolic Syndrome and Its Components. Endocr Metab Immune Disord Drug Targets. 2017; 17(1):56-62.

11 Reinehr T, Isa A, de Sousa G, Dieffenbach R, Andler W. Thyroid hormones and their relation to weight status. Horm Res. 2008;70(1): 51-7.

12 Ochs N, Auer R, Bauer DC, Nanchen D, Gussekloo J, Cornuz J, et al. Meta-analysis: subclinical thyroid dysfunction and the risk for coronary heart disease and mortality. Ann Intern Med. 2008 Jun;148(11):832-45.

13 Bartness TJ, Vaughan CH, Song CK. Sympathetic and sensory innervation of brown adipose tissue. Int J Obes (Lond). 2010 Oct;34 Suppl 1:S36-42.

14 Peirce V, Carobbio S, Vidal-Puig A. The different shades of fat. Nature. 2014 Jun; 510(7503):76-83. 
15 Kajimura S, Spiegelman BM, Seale P. Brown and Beige Fat: Physiological Roles beyond Heat Generation. Cell Metab. 2015 Oct;22(4): 546-59.

16 Rosenwald M, Perdikari A, Rülicke T, Wolfrum C. Bi-directional interconversion of brite and white adipocytes. Nat Cell Biol. 2013 Jun;15(6):659-67.

17 Blüher M, Mantzoros CS. From leptin to other adipokines in health and disease: facts and expectations at the beginning of the 21st century. Metabolism. 2015 Jan;64(1):131-45.

18 Martínez-Sánchez N, Alvarez CV, Fernø J, Nogueiras R, Diéguez C, López M. Hypothalamic effects of thyroid hormones on metabolism. Best Pract Res Clin Endocrinol Metab. 2014 Oct;28(5):703-12.

19 Ribeiro MO, Carvalho SD, Schultz JJ, Chiellini G, Scanlan TS, Bianco AC, et al. Thyroid hormone-sympathetic interaction and adaptive thermogenesis are thyroid hormone receptor isoform-specific. J Clin Invest. 2001 Jul;108(1):97-105.

20 Johann K, Cremer AL, Fischer AW, Heine M, Pensado ER, Resch J, et al. Thyroid-Hormone-Induced Browning of White Adipose Tissue Does Not Contribute to Thermogenesis and Glucose Consumption. Cell Rep. 2019 Jun;27(11):3385-3400.e3.

21 Bianco AC, Sheng XY, Silva JE. Triiodothyronine amplifies norepinephrine stimulation of uncoupling protein gene transcription by a mechanism not requiring protein synthesis. J Biol Chem. 1988 Dec;263(34):18168-75.

22 Silva JE, Larsen PR. Adrenergic activation of triiodothyronine production in brown adipose tissue. Nature. 1983 Oct;305(5936): $712-3$.

23 Christoffolete MA, Linardi CC, de Jesus L, Ebina KN, Carvalho SD, Ribeiro MO, et al. Mice with targeted disruption of the Dio2 gene have cold-induced overexpression of the uncoupling protein 1 gene but fail to increase brown adipose tissue lipogenesis and adaptive thermogenesis. Diabetes. 2004 Mar;53(3): $577-84$.

24 López M, Varela L, Vázquez MJ, RodríguezCuenca S, González CR, Velagapudi VR, et al. Hypothalamic AMPK and fatty acid metabolism mediate thyroid regulation of energy balance. Nat Med. 2010 Sep;16(9):1001-8.

25 Cabral A, Valdivia S, Reynaldo M, Cyr NE, Nillni EA, Perello M. Short-term cold exposure activates TRH neurons exclusively in the hypothalamic paraventricular nucleus and raphe pallidus. Neurosci Lett. 2012 Jun;518(2): 86-91.

26 Nillni EA. Regulation of the hypothalamic thyrotropin releasing hormone (TRH) neuron by neuronal and peripheral inputs. Front Neuroendocrinol. 2010 Apr;31(2):134-56.

27 Nomura E, Toyoda N, Harada A, Nishimura $\mathrm{K}$, Ukita C, Morimoto S, et al. Type 2 iodothyronine deiodinase is expressed in human preadipocytes. Thyroid. 2011 Mar;21(3):305-10.
28 Lee JY, Takahashi N, Yasubuchi M, Kim YI, Hashizaki H, Kim MJ, et al. Triiodothyronine induces UCP-1 expression and mitochondrial biogenesis in human adipocytes. Am J Physiol Cell Physiol. 2012 Jan;302(2):C46372.

29 Lombardi A, Senese R, De Matteis R, Busiello RA, Cioffi F, Goglia F, et al. 3,5-Diiodo-L-thyronine activates brown adipose tissue thermogenesis in hypothyroid rats. PLoS One. 2015 Feb;10(2):e0116498.

30 Senese R, Cioffi F, De Matteis R, Petito G, de Lange P, Silvestri E, et al. 3,5 Diiodo-l-Thyronine $\left(\mathrm{T}_{2}\right)$ Promotes the Browning of White Adipose Tissue in High-Fat Diet-Induced Overweight Male Rats Housed at Thermoneutrality. Cells. 2019 18;8(3):256.

31 Medina-Gomez G, Calvo RM, Obregon MJ. Thermogenic effect of triiodothyroacetic acid at low doses in rat adipose tissue without adverse side effects in the thyroid axis. Am J Physiol Endocrinol Metab. 2008 Apr;294 (4):E688-97.

32 Lin JZ, Martagón AJ, Cimini SL, Gonzalez DD, Tinkey DW, Biter A, et al. Pharmacological Activation of Thyroid Hormone Receptors Elicits a Functional Conversion of White to Brown Fat. Cell Rep. 2015 Nov;13(8):152837.

33 Finan B, Clemmensen C, Zhu Z, Stemmer K, Gauthier K, Müller L, et al. Chemical Hybridization of Glucagon and Thyroid Hormone Optimizes Therapeutic Impact for Metabolic Disease. Cell. 2016 Oct;167(3):843-857.e14.

34 Beaudry JL, Kaur KD, Varin EM, Baggio LL, Cao X, Mulvihill EE, et al. The brown adipose tissue glucagon receptor is functional but not essential for control of energy homeostasis in mice. Mol Metab. 2019 Apr;22:37-48.

35 Weiner J, Kranz M, Klöting N, Kunath A, Steinhoff K, Rijntjes E, et al. Thyroid hormone status defines brown adipose tissue activity and browning of white adipose tissues in mice. Sci Rep. 2016 Dec;6(1):38124.

36 Fontaine DA, Davis DB. Attention to Background Strain Is Essential for Metabolic Research: C57BL/6 and the International Knockout Mouse Consortium. Diabetes. 2016 Jan;65(1):25-33.

37 Seale P, Conroe HM, Estall J, Kajimura S, Frontini A, Ishibashi J, et al. Prdm16 determines the thermogenic program of subcutaneous white adipose tissue in mice. J Clin Invest. 2011 Jan;121(1):96-105.

38 van der Stelt I, Hoevenaars F, Široká J, de Ronde L, Friedecký D, Keijer J, et al. Metabolic Response of Visceral White Adipose Tissue of Obese Mice Exposed for 5 Days to Human Room Temperature Compared to Mouse Thermoneutrality. Front Physiol. 2017 Mar;8:179.

39 Alvarez-Crespo M, Csikasz RI, MartínezSánchez N, Diéguez C, Cannon B, Nedergaard J, et al. Essential role of UCP1 modulating the central effects of thyroid hormones on energy balance. Mol Metab. 2016 Feb;5(4): 271-82.
40 Gachkar S, Oelkrug R, Martinez-Sanchez N, Rial-Pensado E, Warner A, Hoefig CS, et al. 3-Iodothyronamine Induces Tail Vasodilation Through Central Action in Male Mice. Endocrinology. 2017 Jun;158(6):1977-84.

41 Dittner C, Lindsund E, Cannon B, Nedergaard J. At thermoneutrality, acute thyroxineinduced thermogenesis and pyrexia are independent of UCP1. Mol Metab. 2019 Jul;25: 20-34.

42 Sundin U. GDP binding to rat brown fat mitochondria: effects of thyroxine at different ambient temperatures. Am J Physiol. 1981 Sep;241(3):C134-9.

43 Ramadan W, Marsili A, Larsen PR, Zavacki AM, Silva JE. Type-2 iodothyronine 5'deiodinase (D2) in skeletal muscle of C57Bl/6 mice. II. Evidence for a role of D2 in the hypermetabolism of thyroid hormone receptor a-deficient mice. Endocrinology. 2011 Aug; 152(8):3093-102.

44 Nillni EA, Vaslet C, Harris M, Hollenberg A, Bjørbak C, Flier JS. Leptin regulates prothyrotropin-releasing hormone biosynthesis. Evidence for direct and indirect pathways. J Biol Chem. 2000 Nov;275(46):36124-33.

45 Harris M, Aschkenasi C, Elias CF, Chandrankunnel A, Nillni EA, Bjøorbaek C, et al. Transcriptional regulation of the thyrotropin-releasing hormone gene by leptin and melanocortin signaling. J Clin Invest. 2001 Jan;107(1):111-20.

46 Perello M, Cakir I, Cyr NE, Romero A, Stuart RC, Chiappini F, et al. Maintenance of the thyroid axis during diet-induced obesity in rodents is controlled at the central level. Am J Physiol Endocrinol Metab. 2010 Dec;299 (6):E976-89.

47 Farooqi IS, Matarese G, Lord GM, Keogh JM, Lawrence E, Agwu C, et al. Beneficial effects of leptin on obesity, $\mathrm{T}$ cell hyporesponsiveness, and neuroendocrine/metabolic dysfunction of human congenital leptin deficiency. J Clin Invest. 2002 Oct;110(8):1093-103.

48 Mantzoros CS, Ozata M, Negrao AB, Suchard MA, Ziotopoulou M, Caglayan S, et al. Synchronicity of frequently sampled thyrotropin (TSH) and leptin concentrations in healthy adults and leptin-deficient subjects: evidence for possible partial TSH regulation by leptin in humans. JClin Endocrinol Metab. $2001 \mathrm{Jul}$; 86(7):3284-91.

49 Syed MA, Thompson MP, Pachucki J, Burmeister LA. The effect of thyroid hormone on size of fat depots accounts for most of the changes in leptin mRNA and serum levels in the rat. Thyroid. 1999 May;9(5):503-12.

50 Menendez C, Baldelli R, Camiña JP, Escudero B, Peino R, Dieguez C, et al. TSH stimulates leptin secretion by a direct effect on adipocytes. J Endocrinol. 2003 Jan;176(1):7-12.

51 Santini F, Galli G, Maffei M, Fierabracci P, Pelosini C, Marsili A, et al. Acute exogenous TSH administration stimulates leptin secretion in vivo. Eur J Endocrinol. $2010 \mathrm{Jul}$; 163(1):63-7. 
52 Erickson DZ, Harteneck DA, Erickson BJ, Dutton CM, Bahn RS. Induction of leptin expression in orbital preadipocyte fibroblasts. Thyroid. 2001 Mar;11(3):221-6.

53 Fisher FM, Kleiner S, Douris N, Fox EC, Mepani RJ, Verdeguer F, et al. FGF21 regulates PGC- $1 \alpha$ and browning of white adipose tissues in adaptive thermogenesis. Genes Dev. 2012 Feb;26(3):271-81.

54 Adams AC, Astapova I, Fisher FM, Badman MK, Kurgansky KE, Flier JS, et al. Thyroid hormone regulates hepatic expression of fibroblast growth factor 21 in a PPARalphadependent manner. J Biol Chem. 2010 May; 285(19):14078-82.
55 Shu L, Hoo RLC, Wu X, Pan Y, Lee IPC, Cheong LY, et al. A-FABP mediates adaptive thermogenesis by promoting intracellular activation of thyroid hormones in brown adipocytes. Nat Commun. 2017 Jan; 8:14147.

56 Miao Y, Wu W, Dai Y, Maneix L, Huang B, Warner $\mathrm{M}$, et al. Liver $\mathrm{X}$ receptor $\beta$ controls thyroid hormone feedback in the brain and regulates browning of subcutaneous white adipose tissue. Proc Natl Acad Sci USA. 2015 Nov;112(45):14006-11.

57 Wang H, Zhang Y, Yehuda-Shnaidman E, Medvedev AV, Kumar N, Daniel KW, et al. Liver $\mathrm{X}$ receptor alpha is a transcriptional repressor of the uncoupling protein 1 gene and the brown fat phenotype. Mol Cell Biol. 2008 Apr;28(7):2187-200.
58 Chen KY, Cypess AM, Laughlin MR, Haft CR, Hu HH, Bredella MA, et al. Brown Adipose Reporting Criteria in Imaging STudies (BARCIST 1.0): Recommendations for Standardized FDG-PET/CT Experiments in $\mathrm{Hu}-$ mans. Cell Metab. 2016 Aug;24(2):210-22.

59 Hankir MK, Klingenspor M. Brown adipocyte glucose metabolism: a heated subject. EMBO Rep. 2018 Sep;19(9):e46404.

60 Bauwens M, Wierts R, van Royen B, Bucerius J, Backes W, Mottaghy F, et al. Molecular imaging of brown adipose tissue in health and disease. Eur J Nucl Med Mol Imaging. 2014 Apr;41(4):776-91.

61 Krause K. Novel Aspects of White Adipose Tissue Browning by Thyroid Hormones. Exp Clin Endocrinol Diabetes. 2020 Jun;128(607):446-449. 